\title{
Measuring the Complexity of EMG Signal by Using Fuzzy Approximate Entropy in Post-Stroke Patients Rehabilitation
}

\author{
Riries Rulaningtyas ${ }^{1, *}$, Angga Bagus Prasetyo ${ }^{1}$, Akif Rahmatillah ${ }^{1}$, Alfian Pramudita Putra ${ }^{1}$, Osmalina \\ Nur Rahma ${ }^{1}$, Khusnul Ain ${ }^{1}$ and I Putu Alit Pawana ${ }^{2}$ \\ ${ }^{I}$ Biomedical Engineering Study Program, Department of Physics, Faculty of Science and Technology, Universitas Airlangga, Surabaya, \\ Indonesia \\ ${ }^{2}$ Department of Physical Medicine and Rehabilitation, School of Medicine, Universitas Airlangga, Dr. Soetomo General Academic \\ Hospital, Surabaya,Indonesia.
}

Received 18 January 2020; Accepted 23 May 2021

\begin{abstract}
Stroke is one of world's highest medical cases that cause physical or mental problem, even death. The treatment for overcoming stroke so far was by conducting rehabilitation or therapy. The therapy progress could be seen over the improvement indicated by stroke patients after participation in rehabilitation program. So far, the assessment of the level of development exhibited by stroke patients are still subjective. Meanwhile, Electromyograph (EMG) can be used as a quantitative method to evaluate the patient's improvement. The complexity of the EMG signal could define the progress of a rehabilitation process related to muscle function. One of the methods to calculate the complexity is fuzzy approximate entropy (fApEn). This study aims to propose fApEn as a quantitative method to evaluate the condition of stroke patients by comparing the complexity of the normal EMG, affected, and unaffected side limb of the patient's EMG signal. The value of fApEn on Subject 1 showed that the affected side obtained the complexity of 0.18141 and on the unaffected side of 0.80740 . Subject 2 showed the complexity on the affected side of 0.58597 and the unaffected side of 1.02787 , whereas the healthy subjects showed the complexity of 2.07856. The statistical results showed that the data were homogeneous and normally distributed. There was a significant difference between the results of fApEn on the affected side and the unaffected side. Thus, fApEn could be used as a quantitative method to determine the condition of muscle activity.
\end{abstract}

Keywords: Electromyography, Fuzzy Approximate Entropy, Rehabilitation, Stroke

\section{Introduction}

Stroke is the most significant cause of death and disability. It occurs in almost all hospitals. The disability experienced by stroke sufferers is caused by organ disorders or organ dysfunction such as hemiparesis, aphasia and dysarthria, and cognitive disorders [1-2]. All of these things will result in disability, which includes an inability to walk, communicate, and self-care inability [3]. Based on the study of Haley et al. (2013), it was mentioned that the effect caused by stroke also affected the family and surrounding society, besides affecting the patients themselves [4]. The percentage of the increase in patients with stroke each year even step up with the rise of age based on the study of Basic Health Research in Indonesia in 2013. The highest case occurred in the patient with the age of 75 years old or above, which was $43.1 \%$, and the lowest incident happened in the patients age between 15 and 24 years old, which was $0.2 \%$. Among them, $7.1 \%$ were men, and $6.8 \%$ were women [5].

The treatment method that has been used so far to restore the function of organs damaged by stroke is to carry out a rehabilitation program. A rehabilitation program is an act of health service by taking a medical, psychosocial, and educational-vocational approach. It involves a multidisciplinary approach consisting of neurologists, medical rehabilitation doctors, nurses, medical social

\footnotetext{
*E-mail address: riries-r@fst.unair.ac.id

ISSN: 1791-2377 @ 2021 School of Science, IHU. All rights reserved. doi:10.25103/jestr.143.10
}

workers, physiotherapists, occupational therapists, psychologists, and clients and families who also played a role $[3,6]$. The success of a series of rehabilitation programs is indicated by the level of improvement done by stroke patients after the rehabilitation program. One method for assessing the level of development so far has been carried out by the FuglMeyer way and the Manual Muscle Test (MMT) [7,8]. FuglMeyer and MMT are used to assess motor function in stroke patients before and after getting a rehabilitation program [7]. Data pattern repetition of EMG signals from stroke patients could be used to improve functional recovery $[9,10]$. The repetition pattern in the EMG is also used to predict the movements that will be carried out by stroke sufferers. The research was also supported by research conducted by Sun et al. (2014) [3]. The study used EMG signal data for stroke patients before and after the rehabilitation program was given, the EMG data was then analyzed using Fuzzy Approximate Entropy (fApEn). This method could determine the complexity in the EMG signal segment by using a fuzzy concept. The complexity of EMG could give some information regarding the change in the neuromuscular system. This method has been widely used in several biosignals and clinical datasets and indicated as an excellent method compared to the other complexity measurement process [11-13]. Based on those studies, we were motivated to implement the fApEn method in EMG signal data from patients with stroke on both sides of the hand, which represented the affected side and the unaffected side. Besides that, both data would be compared to a healthy subject. In the 
end, we could recommend a new method to classify the level of muscle repair based on the results of the calculations of the Fuzzy Approximate Entropy method.

\section{Materials and Methods}

\subsection{Subjects and Experimental Procedure}

Two post-stroke subjects with the same characteristics, namely paralysis in the upper limb and one healthy Subject as a control. Both post-stroke subjects were female with age of 58 y.o. and 47 y.o., respectively. They had paralysis on the right side of the body. The purpose of testing the Subject is to obtain quantitative values of changes in EMG signals on the affected side and unaffected side of the patient's hands, which were then associated with physiological symptoms to the muscles that are known using the Fuzzy Approximate Entropy method. Retrieval of EMG data in each Subject used $\mathrm{Ag} / \mathrm{AgCl}$ electrodes associated with EMG Shield OLIMEX. It was expected that by using these methods, the accurate results could be obtained and can illustrate the increase or decrease in the level of complexity of the muscle signal that has been measured using only qualitative questionnaires. All results received both the data collected on the affected side and the unaffected side, as well as the subject data under normal conditions, is then recorded. The proposed method was depicted as block diagram in Fig. 1. The fApEn process was shown in Fig. 2.

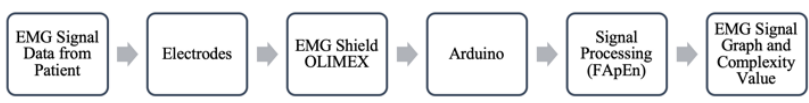

Fig. 1. The Block Diagram of Proposed Method to Evaluate the EMG Signal of Stroke Patient Based on the Complexity

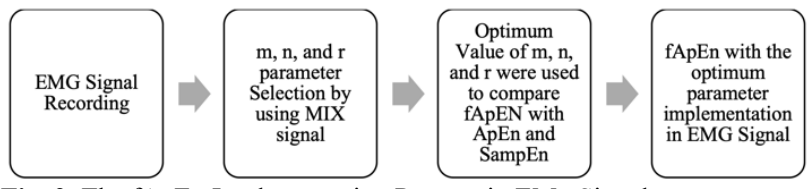

Fig. 2. The fApEn Implementation Process in EMg Signal

\subsection{Fuzzy Approximate Entropy}

This study used the third order of the Besselworth filter to process EMG data and eliminate the noise. After the EMG signal has been successfully recorded, the EMG raw data was stored at a sampling frequency of $100 \mathrm{data} / \mathrm{s}$. The stored data was then calculated its complexity value by using the preprogrammed $f A p E n$ method. The calculation of the FApEn from the series number of the sample $(\mathrm{N})$ was performed based on the study of Rui Sun et al. in 2014 [3].

First, for given integers (m), vector sequences with a dimension of $\mathrm{m}$ are formed and shown in Equation 1.

$$
\begin{aligned}
& A_{i}^{m}=\{a(i), \ldots, a(i+m-1)\}-\frac{1}{m \sum_{j=0}^{m-1} a(i+j)} \\
& a_{0}(i)=\frac{1}{m \sum_{j=0}^{m-1} a(i+j) .}
\end{aligned}
$$

For each $A_{i}^{m}$, the distance $d_{i j}^{m}$ of two vectors $A_{i}^{m}$ and $A_{j}^{m}$ is shown in Equation 3.

$$
\begin{aligned}
& d_{i j}^{m}=\max _{k \in(0, m-1)} \mid a(i+k)-a_{0}(j)-a(j+k)- \\
& \frac{1}{m} \sum_{j=0}^{m-1} a(i+j) \mid
\end{aligned}
$$

The similarity degree of two vectors $A_{i}^{m}$ and $A_{j}^{m}$ is shown in Equation 4.

$$
D_{i j}=\exp \left(-\left(\frac{d_{i j}^{m}}{r}\right)^{n}\right)
$$

where $r$ is the width of the boundary and $n$ is the gradient of the boundary.

The similarity degree was then averaged as a function $\varphi^{m}$ and is defined as shown in Equation 5.

$$
\varphi^{m}(n, r)=\frac{1}{N-m+1} \sum_{i=1}^{N-m+1} \ln \left(\frac{1}{N-m+1} \sum_{i=1}^{N-m+1} D_{i j}^{m}\right)
$$

The $f A p E n$ is then calculated as shown in Equation 6.

$$
f \operatorname{ApEn}(m, n, r, N)=\varphi^{m}(n, r)-\varphi^{m+1}(n, r)
$$

\subsection{Parameter Selection and Simulation Test}

Some simulated signals were created to evaluate the algorithm of the fuzzy approximate entropy. That signal input was built based on the study of Pincus (1991), which states that signal input with different complexity could be built based on Equation 7 [14].

$$
\operatorname{MIX}(p)_{j}=\left(1-M_{j}\right) K_{j}+M_{j} L_{j} \quad(1 \leq j \leq N)
$$

Where $K_{j}=\sqrt{2} \sin (2 \pi j / 12), L_{j}$ was a set of Independent Identically Distributed (i.i.d.) real random variables with a uniform distribution within $[-\sqrt{3}, \sqrt{3}]$, and $M_{j}$ were i.i.d. random variable, where $M_{j}=1$ had a probability of $p$ and $M_{j}=0$ had a probability of $1-p$. The increase of $p$ value indicated the rise of randomness and complexity in the $\operatorname{MIX}(p)$ process and led to higher entropy values. The simulated signals with different complexity were shown in Fig. 3.

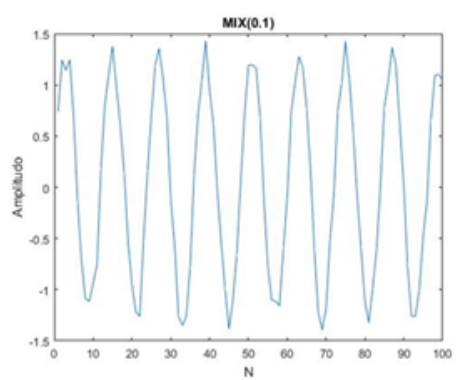

(a)

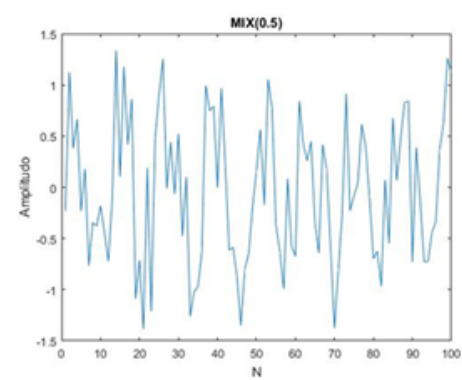

(b)

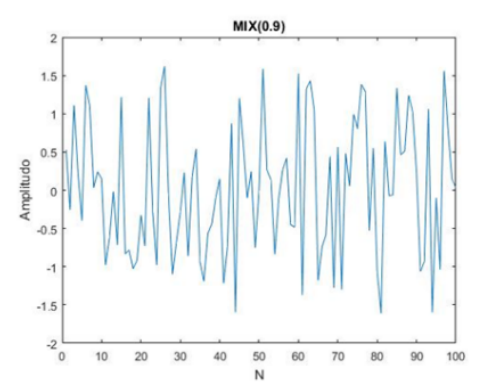

(c)

Fig. 3. The generated Signal with Several probability which represents the amount of randomness in the signal (a) 0.1 , (b) 0.5 , and (c) 0.9 
Besides the simulated signals by using the same methods by Pincus (1991), Gaussian noise and a chirp signal were used in this study to evaluate the performance of the fApEn algorithm [14]. Those signals were also compared with the normal EMG signals.

\subsection{Comparison of fApEn and The Other Entropy-Based Method}

After obtaining the optimum value of $n, m$, and $r$, the fApEn method was compared with the other entropy-based method to evaluate the complexity, which was Approximate Entropy (ApEn) and Sample Entropy (SampEn). Both methods were mainly used to evaluate complexity in the signals. The ApEn method has been calculated the complexity by using Equation $8-13$. For the $\mathrm{N}$ time series of data $\{a(i): 1 \leq i \leq N\}$ with vector $x(1)$ is going through $x(N-m+1)$, the processing data was shown in Equation 8 [13].

$x(i)=\{a(i), a(i+1), \ldots, a(i+m-1)\}$.

$x(i)$ includes $m$ value, respectively. $m$ is the window length in the data sequencing process. The vector $\{x(i), 1 \leq$ $i \leq N-m+1\}$, was generalized by subtracting the mean value $q 0(i)$, as shown in Equation 9.

$Q(i)=\{a(i)-q 0(i), a(i+1)-q 0(i), \ldots, a(i+m-1)-$ $q 0(i)\}$

$Q(i)=\{q(i), q(i+1), \ldots, q(i+m-1)\}$

With $q 0(i)=\frac{\sum_{j=0}^{m-1} a(i+j)}{m}$.

The distance of $Q(i)$ and $Q(j)$ was defined as $d_{i j}$ and calculated, as shown in Equation 10.

$d_{i j}=\max _{k \in(0, m-1)}|q(i+k)-q(j+k)|$

By using the tolerance $r$, the $C_{i}^{m}(r)$ could be defined for each $i$ within $N-m+1$, as shown in Equation 11 .

$C_{i}^{m}(r)=\frac{\sum j\left((j \leq N-m+1) \text { and } d_{i j} \leq r\right)}{N-m+1}$

The $\ln$ of $C(r)$ was then averaged over I, as shown in Equation 12.

$\emptyset^{m}(r)=\frac{1}{N-m+1} \sum_{i=1}^{N-m+1} \ln C_{i}^{m}(r)$

The ApEn was estimated, as shown in Equation 13.

$\operatorname{ApEn}(m, r, N)=\emptyset^{m}(r)-\emptyset^{m+1}(r)$

The SampEn method was a developed version of the ApEn method, which did not include the self-matched vector. By introducing this step, there would be no limitation in terms of data length, and it showed consistency in several conditions. Define $S_{i}^{m}(r)$ and $T_{i}^{m}(r)$, which were the same way as $C_{i}^{m}(r)$ but excluding the self-matching process by omitting the same value of $i$ and $j$ while $j$ ranges from 1 to $N-m . S_{i}^{m}(r)$ represents the vector $X_{j}^{m}$ within $\mathrm{r}$ of $X_{i}^{m}$ while $T_{i}^{m}(r)$ represents the vector $X_{j}^{m+1}$ within $\mathrm{r}$ of $X_{i}^{m+1}$ Both $S_{i}^{m}(r)$ and $T_{i}^{m}(r)$ were shown in Equation 14 and 15 [13].

$S^{m}(r)=\frac{1}{N-m} \sum_{i=1}^{N-m} S_{i}^{m}(r)$ or $T^{m}(r)=\frac{1}{N-m} \sum_{i=1}^{N-m} S_{i}^{m}(r)$
$\operatorname{SampEn}(m, r, N)=-\ln \left[\frac{s^{m}(r)}{T^{m}(r)}\right]$

\subsection{Data Analysis}

The normality of the sample was tested to determine the normal distribution of the variable value distribution. The variables tested in this case were independent. The Kolmogorov-Smirnov significance score was used to evaluate the normality of the sample. After the normality test, the homogeneity sample was then tested using the Levene test.

The data that has been tested with normality and homogeneity test showed that they were normally distributed and homogeneous, then the T-Test was performed. T-Test was used to see whether there are differences between the two data processed variables, namely the data on the affected side and the unaffected side. If the tested data were declared not normally distributed, then it was proceeded to the MannWhitney test to find out the differences between the two data from the processed variables.

\section{Results}

\subsection{Parameter Selection of fApEn}

Several essential parameters in measuring complexity are relative consistency, monotonicity, and continuity. Relative consistency is the condition if a signal has more complexity than the others, then the entropy value would be higher too. Monotonicity represents a repetitious cycle when some parameters of the signal are changed. Continuity means that the signal should be stable and no significant change when there is a small change in some settings in the signal. The performance of the fApEn method, which reflects the relative consistency, monotonicity, and continuity, was evaluated by using the simulated signals and several signals, such as white noise, chirp signal, and standard EMG signal [3]. Those three parameters were used to select the best parameters $(\mathrm{m}, \mathrm{N}, \mathrm{r})$ to be used in the EMG signal processing.

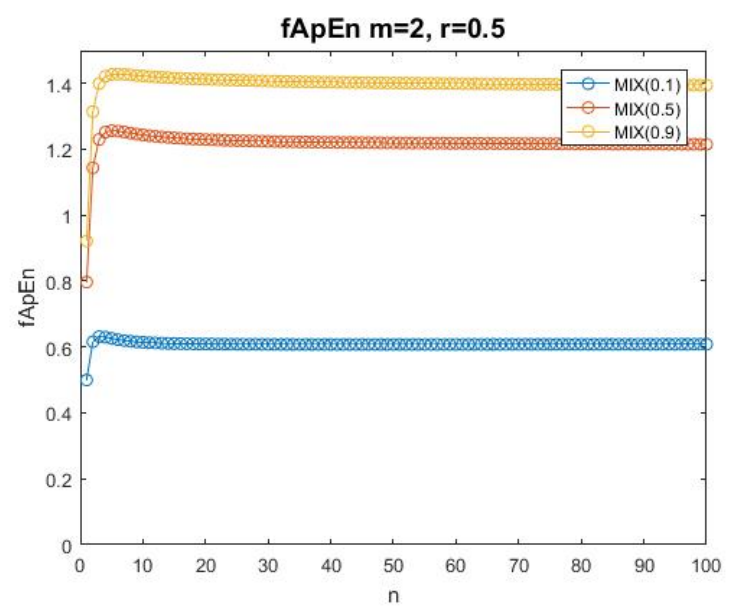

Fig.4. $f A p E n$ results with variations in the value of $n$ between $0-100$

Based on Fig. 4 and Fig. 5, the result of this study was consistent with research conducted by Chen et al. (2006, 2009), and Ao et al. (2015) by using MIX with $\mathrm{p}=0.1,0.5$, and 0.9 input signals, where the $f A p E n$ calculation result of MIX (0.9) had the highest fApEn value and followed by MIX (0.5) and MIX (0.1) with the lowest fApEn value [3, 12-13]. Fig. 4 also indicated a trend of the rapid increase of $f A p E n$ when the value of $\mathrm{n}$ ranges between 1 to 5 , and $f A p E n$ reached 
a stable point when the value of $\mathrm{n}$ was more significant than 5. The value of $f A p E n$ would change tended to decrease with increasing values of the parameter $r$ that varied from 0.1 to 1 with every step of 0.02 . A rapid decrease was found in the fApEn value if a small $r$ (less than 0.1 ) value was used, as $s$ shown in Fig. 5.

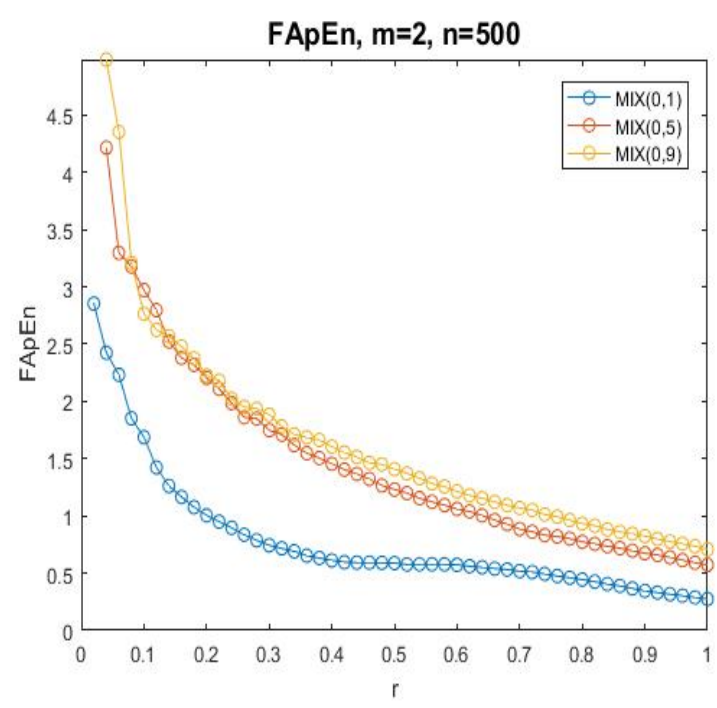

Fig. 5. $f A p E n$ results with variations in the value of $r$ between $0-1$

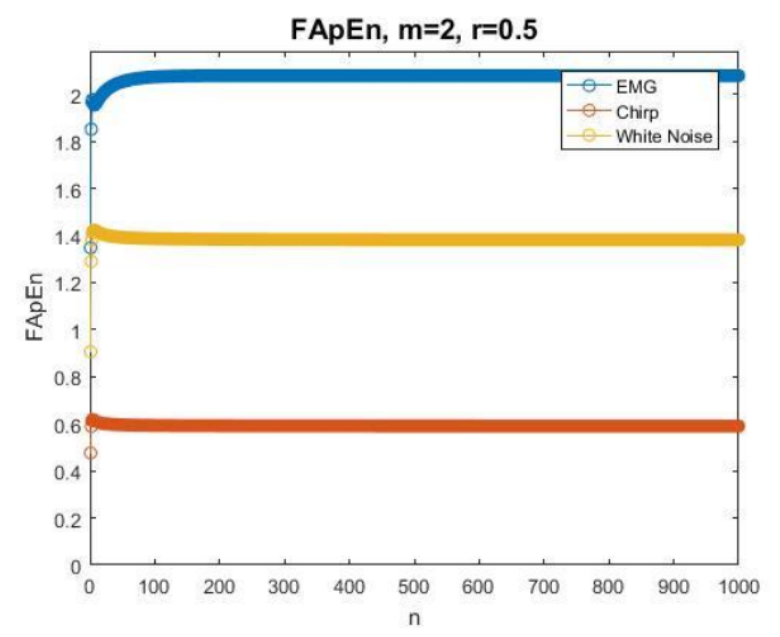

Fig. 6. fApEn value of EMG, chirp signals and white noise with variations in the value of $n$ between $0-100$

After testing the algorithm by using the simulated signals MIX (p), the algorithm was also tested by using a chirp signal, white noise, and standard EMG signal. The result was shown in Fig. 6 and Fig. 7. A chirp signal is a signal with an ascending frequency (up-chirp) or descending frequency (down-chirp) in the time domain with a regular sinusoidal shape and a low complexity. In contrast, a white noise signal is a random signal with a similar intensity and different frequency so that it gives a constant power spectral density.

Based on the definition of both signals, they should have different fApEn value. EMG signal should have the highest fApEN value compared to the other two signals. The result in Fig. 6 and Fig. 7 showed that with several values of $n$ and $r$, the standard EMG signal had the highest fApEn value, followed by the white noise signal and chirp signal. Fig. 7 with a variation of $r$ value in the fApEn calculation process showed an inconsistent result in the low value of $r$. This result was suited with the study of Chen et al. (2007) and Ao et al. (2015) which mentioned that the best value to evaluate the complexity of EMG signals by using fApEn method was $\mathrm{m}=$ $2, \mathrm{n}=500$, and $\mathrm{r}=0.5[3,12-13]$. Thus, these values were used to evaluate the post-stroke patients' EMG signal in term of the complexity.

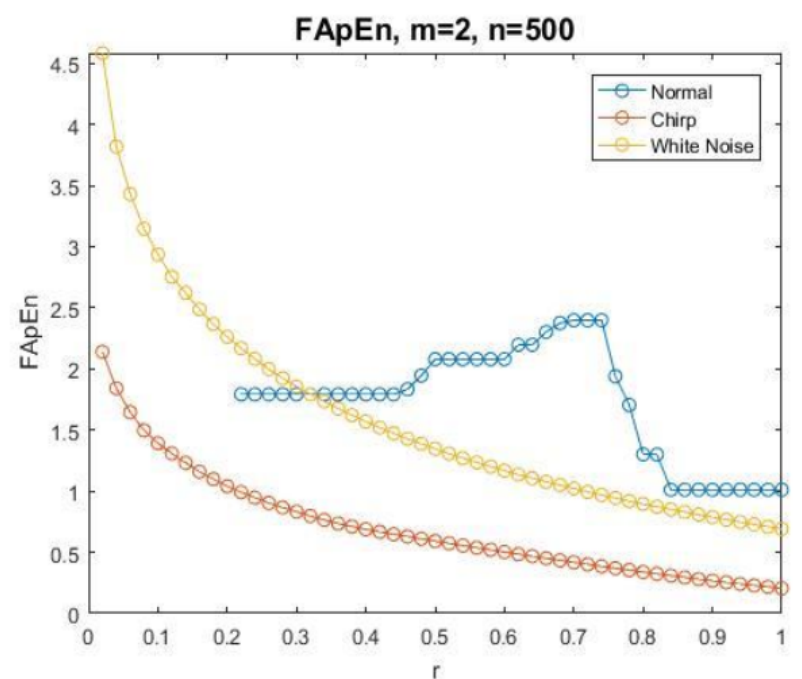

Fig. 7. fApEn value of EMG, chirp signals, and white noise with variations in the value of $r$ between $0-1$.

\subsection{Comparison of fApEn and The Other Entropy-Based Method}

The result of parameter selection was then implemented in three different methods to evaluate the complexity of the signal based on the entropy, which was ApEn, SampEn, and fApEn. The tested signal was the signal used for the parameter selection, which was a simulated signal called MIX with a changeable probability of complexity called $p$. The result of ApEn and SampEn were shown in Fig. 8 and 9. The value of ApEn and SampEn was increasing while the probability value $p$ increased.

Fig. 8 and 9 showed the result of ApEn and SampEn calculation in three signals with different complexity. There was a crossover in Figure 6 and 7 with a high value of $r$ (more than 0.5), while it could not be found in Fig. 5. The result of fApEn in Figure 3 showed the best monotonicity, consistency, and continuity among the tested methods. The result of SampEn and ApEn showed some fluctuations in the higher value of $r$. The fApEn also showed a more continuous and smoother change compared to the others.

Moreover, a significant fluctuation and discontinuity were shown in the result of SampEn and ApEn with a small change in the value of $r$. This result was correlated with the study of Chen et al. (2009), which also mentioned the same behavior [12]. Those three parameters showed the role of entropy to determine the complexity. Based on this comparison, fApEn showed a superior result in terms of monotonicity, consistency, and continuity compared to SampEn and ApEn. It meant that it was more suitable to be used to evaluate the complexity of the EMG signal since it was the random signal which should be more complex [12,13].

Based on the parameter selection, relative consistency of the fApEn value was depicted in Figure 4 and 5 with $n$ more than 5 and $r$ more than 0.3 . The fApEn value was more consistent at those parameters value compared to Figure 8 and 9 when ApEn and SampEn were used. ApEn showed a poor consistency with $\mathrm{r}$ value less than 0.2 or more than 0.6 . 
Besides that, in term of monotonicity and continuity, ApEn and SampEn was limited to the value of $\mathrm{r}[12,13]$.



Fig. 8. ApEn results with variations in the value of $\mathrm{r}$ between $0-1$



Fig. 9. SampEn results with changes in the value of $\mathrm{r}$ between $0-1$

\subsection{Performance on EMG Signals}

Based on the results of Fig. 10, the value of $f A p E n$ on the affected side on Subject 1 obtained a smaller result compared to the $f A p E n$ on the unaffected side, which was 0.18141 and 0.80740 , respectively. Fig. 11 showed the value of $f A p E n$ on the affected side of the subject 2 in smaller results than the value of $f A p E n$ on the unaffected side, which was 0.58597 and 1.02787, respectively. The result in each Subject 1 and 2 were similar. Namely, the value of $f A p E n$ on the affected side was smaller than the unaffected side. A little FApEn value indicates that there is a low value for the complexity of the muscle signal and an increase in regularity in the muscle signal. The signal amplitude on the affected side was smaller when compared to the signal amplitude on the unaffected side. This difference in amplitude also showed that the EMG signal was affected by the stroke condition of the subject.

When the value of fApEn in Subject 1 and 2 was compared to the value of $f A p E n$ in standard EMG signal shown in Figure 10 and 11, the value of $f A p E n$ on the unaffected side was smaller than that of standard EMG signal. These results were supported by a study conducted by Ao et al. (2015), which stated that there was a decrease in value or deficit on the unaffected side [3]. It was caused by strokes that occur on the unaffected side and the affected side. The density of muscle fibers on the unaffected side in post-stroke patients is relatively smaller compared to the muscle fibers in healthy people. Besides that, the presence of lesions in half the body could have an impact on the subcortical structures which affected the motor control and led to a disruption in nerve pathways in the unaffected side of the body [15]. The decrease in the values of $f A p E n$ on both sides was caused by a stroke. It was an indicator that there was a reduction in the number of firing rate of the motor unit due to the denervation of the muscular, nervous system in that part of the body [16].

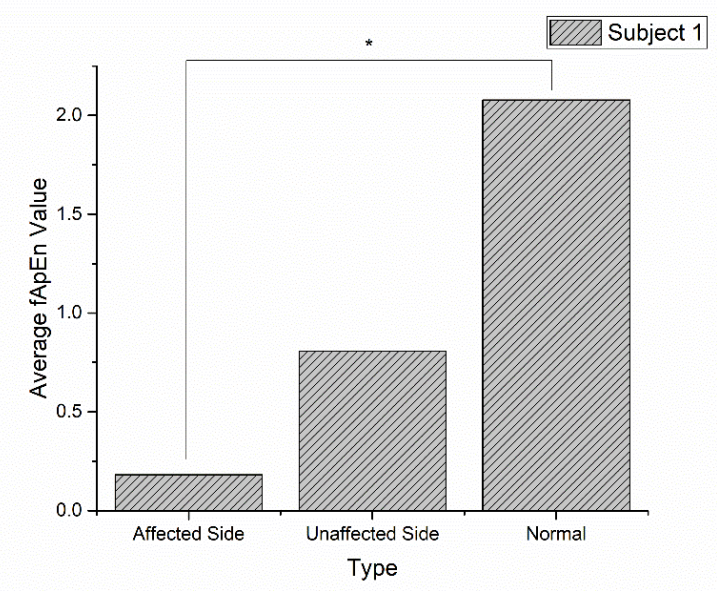

Fig.10. The average value of $f A p E n$ on the Unaffected Side and Affected Side of the 1st Subject Compared to the Normal Subject

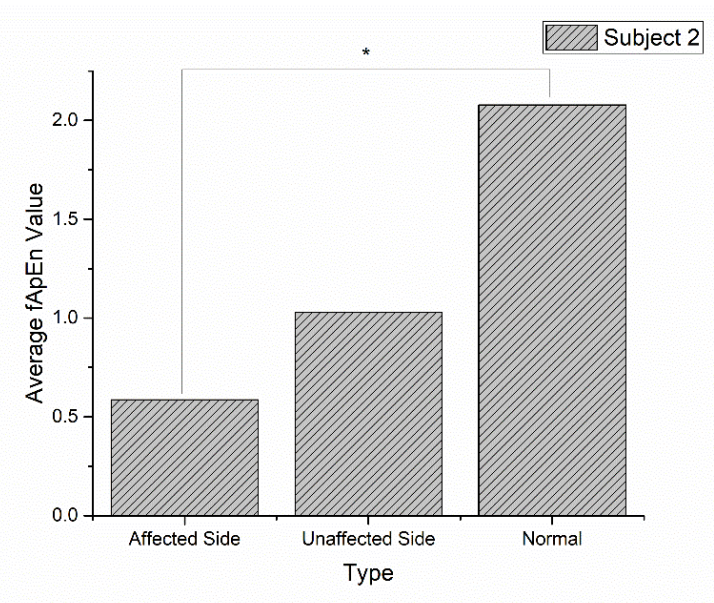

Fig. 11. The average value of $f A p E n$ on Unaffected Side and Affected Side of Subject 2 Compared to Normal Subjects

The normality test is carried out to determine the normal distribution of the variable values of the tested data. In this study, the verified data are $f A p E n$ data from the affected side and the unaffected side. In this test, the results show that the affected and unaffected side data are normally distributed. This result is indicated by the significant value that is more than the $p$-value $(0.05)$, namely 0.668 on the affected side and 0.277 on the unaffected side. So that, the assumptions were met, and the test carried out next was the Levene test and TTest to find out the homogeneity of the data and whether there were differences between the two variable data being processed.

Levene Test and T-Test were done after the normality test results and showed that the tested data were normally 
distributed. In the test results, the data had homogeneous variants with a significance value of $0.149(\alpha>0.05)$. Whereas in the T-Test, the Equal Variances Assumed was chosen because of the assumed homogeneity assumptions, which obtained a significance value of $0.002(\alpha<0.05)$. Based on the results of T-Test, there was a difference between the results of the $f A p E n$ on the affected side and the unaffected side.

In this study, Surface Electromyography (sEMG) was used because it was practical and non-invasive. Based on the results obtained above, the $f A p E n$ analysis of the sEMG signal had the potential to be applicable in conducting clinical evaluations of neurological changes caused by stroke. This quantitative measurement was useful for Subject after stroke in observing progress or the success of rehabilitation therapy to improve the treatment by finding the value of $f A p E n$ from each part of the body. However, for this application, a future study is needed on the relationship between clinical scale and $f A p E n$ values with larger sample size. It also requires a usable interface or instructions so that it would be easy to use for calculation and clinical application.

\section{Conclusion}

In this study, the fApEn method could distinguish the complexity value of the input signal with three levels of complexity, namely low (MIX (0.1)), moderate (MIX (0.5)), and high (MIX (0.9)). The parameter values $\mathrm{m}=2, \mathrm{n}=500$, and $\mathrm{r}=0.5$ were proposed because they were the most optimal values to be used in the fApEn method shown by the use in white noise, chirp signal, and standard EMG signal. There was a significant difference between the results of fApEn on the affected side and the unaffected side in both subjects, which indicated that fApEn could be used as a quantitative method to determine the condition of muscle activity in the stroke patients. For future studies, the approach should be tested in real-time conditions to increase the usability and help the medical practitioners to determine the status of stroke patients.

\section{Acknowledgement}

The author would like to our gratitude to the Directorate of Research and Community Development (DRPM) of the Republic of Indonesia through the PTUPT scheme in 2019 with grant number [6/E/KPT/2019].

This is an Open Access article distributed under the terms of the Creative Commons Attribution License.

\section{References}

1. J. Klebic, N. Salihovic, R. Softic, and D. Salihovic, Aphasia disorders outcome after stroke., Med. Arh., 65, 5, p. 283-286 (2011).

2. Y. Bejot et al., One-year survival of demented stroke patients: data from the Dijon Stroke Registry, France (1985-2008)., Eur. J. Neurol., 19, 5, p. 712-717, (2012).

3. D. Ao, R. Sun, K.-Y. Tong, and R. Song, Characterization of strokeand aging-related changes in the complexity of EMG signals during tracking tasks., Ann. Biomed. Eng., 43, 4, p. 990-1002, (2015).

4. W. E. Haley, D. L. Roth, M. Hovater, and O. J. Clay, "Long-term impact of stroke on family caregiver well-being: A population-based case-control study," Proc. ASME Des. Eng. Tech. Conf., pp. 1232$1239,(2015)$.

5. Indonesia Ministry of Health, "RISET KESEHATAN DASAR," Jakarta, (2013).

6. R. Sun, R. Song, and K. Tong, Complexity Analysis of EMG Signals for Patients After Stroke During Robot-Aided Rehabilitation, IEEE Trans. Neural Syst. Rehabil. Eng., 22, 5, p. 1013-1019 (2014).

7. B. Singer and J. Garcia-Vega, The Fugl-Meyer Upper Extremity Scale., J. Physiother., 63, 1, p. 53, (2017).

8. K. M. Conable and A. L. Rosner, A narrative review of manual muscle testing and implications for muscle testing research., J. Chiropr. Med., 10, 3, p. 157-165, (2011).

9. A. Rahmatillah, O. N. Rahma, M. Amin, S. I. Wicaksana, K. Ain, and R. Rulaningtyas, Post-Stroke Rehabilitation Exosceleton
Movement Control using EMG Signal, Int. J. Adv. Sci. Eng. Inf. Technol., 8, 2, p. 616-621, (2018).

10. A. Rahmatillah and A. A. Karimasari, Prototype of Arm Therapy Device Using Myoelectric Signal, in 2017 International Seminar on Sensor, Instrumentation, Measurement and Metrology (ISSIMM) Surabaya, Indonesia, August 25th - 26th, 2017, p. 21-25 (2017).

11. Y. Chen et al., Stroke-Related Changes in the Complexity of Muscle Activation during Obstacle Crossing Using Fuzzy Approximate Entropy Analysis, Front. Neurol., 9, March, (2018).

12. W. Chen, J. Zhuang, W. Yu, and Z. Wang, Measuring complexity using FuzzyEn, ApEn, and SampEn., Med. Eng. Phys., 31, 1, p. 6168, (2009).

13. W. Chen, Z. Wang, and X. Ren, Characterization of surface EMG signals using improved approximate entropy., J. Zhejiang Univ. Sci. B, 7, 10, p. 844-848, (2006).

14. S. M. Pincus, Approximate entropy as a measure of system complexity., Proc. Natl. Acad. Sci. U. S. A., 88, 6, p. 2297-2301, (1991).

15. M. Lukacs, L. Vecsei, and S. Beniczky, Large motor units are selectively affected following a stroke., Clin. Neurophysiol., 119, 11, p. 2555-2558, (2008).

16. L. A. C. Kallenberg and H. J. Hermens, Motor unit properties of biceps brachii during dynamic contractions in chronic stroke patients., Muscle Nerve, 43, 1, p. 112-119, (2011). 